\title{
Granular cells as a marker of early amiodarone hepatotoxicity: a pathological and analytical study
}

\author{
N A SHEPHERD, A M DAWSON,* P R CROCKER, D A LEVISON \\ From the Departments of Histopathology and ${ }^{*}$ Gastroenterology, St Bartholomew's Hospital, London
}

SUMMARY Two contrasting cases of amiodarone hepatotoxicity are described. In one, perivenular $\stackrel{\vec{\rho}}{0}$ collections of swollen cells, probably macrophages, with granular cytoplasm in an otherwise nearly normal liver were observed. Transmission electron microscopy showed that the cytoplasmic granu- $\rightarrow$ larity was due to lysosomal bodies of various sizes. X-ray energy and wavelength spectroscopic analysis showed a high iodine content in these lysosomal bodies, good presumptive evidence of the ${ }^{\infty}$ presence of amiodarone or one of its metabolites. In the second case there was a micronodular cirrhosis, and similar granular cells also containing iodine were seen in the fibrous connective tissue. $\vec{\nabla}$ These cases seem to represent the very early and late stages of amiodarone hepatotoxicity. In a을. patient taking this drug aggregates of swollen granular cells may be a sensitive and histo- $\vec{\varphi}$ pathologically useful marker of early amiodarone toxicity.

Amiodarone is an iodinated amphophilic drug that has been available in Europe for the past 20 years; it was originally used for the treatment of angina. ${ }^{1}$ More recently the drug has been found to be very effective in the treatment of supraventricular tachyarrhythmias, including the Wolff-Parkinson-White syndrome, and in ventricular tachyarrhythmias. The drug is associated, however, with an increasingly recognised number of side effects. ${ }^{2}$ Amiodarone toxic effects are seen in the skin, lung, thyroid, peripheral nervous system, muscle, cornea and liver. ${ }^{2}$

The drug accumulates in many organs, and some side effects, especially those affecting the skin and liver, seem to be dose related. ${ }^{2}$ Amiodarone toxicity is thought to be due to the accumulation of the drug in lysosomes where it combines with lysosomal membrane lipid (due to its amphophilic properties) to produce a secondary phospholipidosis. ${ }^{3}$ In the liver the phospholipidosis occurs in hepatocytes, Kupffer cells, bile duct epithelium and endothelial cells. ${ }^{3}$ We report two cases of amiodarone hepatotoxicity. We believe that the histological features of our first case represent the first documentation of the earliest morphological changes of amiodarone hepatotoxicity and that those of the second represent the terminal stage of liver damage associated with amiodarone.

Accepted for publication 4 November 1986

\section{Case reports}

\section{CASE 1}

A 64 year old man had a myocardial infarction in $\overrightarrow{\overrightarrow{0}}$ 1983 , complicated by cardiac arrest and refractory ventricular arrhythmia. There was no relevant medical history and no history of excessive alcohol intake. He was given amiodarone $200 \mathrm{mg}$ three times? a day. Eight months later he complained of right $:-$ upper quadrant discomfort, nausea, anorexia and $\underline{3}$. weight loss, and he ran a low grade fever up to $38^{\circ} \mathrm{C}$. His liver function tests yielded abnormal results, the aspartate aminotransferase (AST) activity being 0 $54 \mathrm{IU} / 1$ (normal 10 to $40 \mathrm{IU} / \mathrm{l}$ ). His smooth muscle and mitochondrial antibodies were negative, the erythrocyte sedimentation rate was raised at $78 \mathrm{~mm} / \mathrm{first}$ hour, and the globulins raised at $37 \mathrm{~g} / 1, \mathrm{~N}$ mainly due to a rise in the $\alpha_{2}$ fraction. The antinuclear $N$ factor was negative. An ultrasound scan of the liver at N that time was normal. He was loath to undergo liver biopsy. The possibility of a collagen disease was considered, and a trial of prednisolone $(20 \mathrm{mg} /$ day $)$ was given. There was initial improvement. The fever and $\stackrel{\mathcal{S}}{+}$ symptoms of nausea and anorexia disappeared, the erythrocyte sedimentation rate fell to $38 \mathrm{~mm} / \mathrm{first}$ hour and the liver function tests became normal.

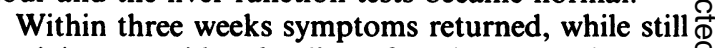
receiving steroids, the liver function tests became $\frac{\varrho}{\sigma}$ abnormal again (AST $141 \mathrm{IU} / \mathrm{l}$ ), and the erythrocyte sedimentation rate rose. At this stage the patient 
underwent a liver biopsy and the prednisolone was stopped. A few days later the symptoms became appreciably worse, with high fever, a generalised dermatitis, and crepitations in the chest. A chest $x$-ray showed basal reticular shadowing typical of amiodarone toxicity. The liver function tests were grossly abnormal, the AST rising to $575 \mathrm{IU} / 1$. The amiodarone was stopped as soon as the rash appeared: prednisolone was started again at a higher dose of $45 \mathrm{mg} /$ day, and over the ensuing weeks he gradually improved. The physical signs in the chest cleared completely and the chest $x$-ray became normal. Within two months of stopping the amiodarone, the liver functions tests had returned to normal.

CASE 2

A 64 year old woman presented in 1977 with the Wolff-Parkinson-White syndrome. There was no relevant medical history and no history of excessive alcohol consumption. There was a strong family history of ischaemic heart disease. She was treated with amiodarone, $200 \mathrm{mg}$ three times a day, diuretics, and $\beta$-blockers. Three years later slate-grey discolouration of her face was noted, a known side effect of amiodarone. $^{2}$ The liver function tests were monitored while the patient was receiving amiodarone. At the time the facial discolouration was noted the AST was raised at $114 \mathrm{IU} / 1$ and the alkaline phsophatase activity (ALP) was $109 \mathrm{IU} / 1$ (normal 25-100 IU/1). Her symptoms, especially palpitations, were difficult to control. The patient, despite warnings of the potential hazards, pleaded to stay on high doses of amiodarone and the drug was continued. Four years after starting the drug she became weak and bedridden and on admission to hospital became jaundiced and developed ascites. Liver function tests at this time showed a bilirubin concentration of $44 \mu \mathrm{mol} / 1$ (normal $2-17 \mu \mathrm{mol} / \mathrm{l}$ ), ALP of $150 \mathrm{IU} / \mathrm{l}$, and AST of $172 \mathrm{IU} / \mathrm{l}$. She developed signs of hepatic encephalopathy, confirmed by electroencephalography. She was also found to have a sensorimotor neuropathy.

Her management consisted of intensive nursing care, nasogastric feeding, and physiotherapy. Four months after admission, however, she developed signs of bronchopneumonia and died. A liver biopsy was not performed in life. A postmortem examination confirmed bronchopneumonia. The liver weighed $1800 \mathrm{~g}$ and showed a micronodular cirrhosis. The brain showed macroscopic and histological changes consistent with hepatic encephalopathy. The heart weighed $370 \mathrm{~g}$ and the coronary arteries were all widely patent. The chambers and valves of the heart seemed to be normal. Histological examination of the heart showed patchy fibrosis of the myocardium with no evidence of past or recent infarction. The thyroid gland was normal on both macroscopic examination and histology.

\section{Material and methods}

The needle biopsy of liver of case 1 and samples of the liver from the necropsy of case 2 were routinely fixed in $10 \%$ buffered formalin and processed in paraffin wax. Four micron sections were stained with haematoxylin and eosin, periodic acid Schiff (PAS), Gordon and Sweets' reticulin, Masson's trichrome, Perls's Prussian blue and van Gieson stains.

Paraffin sections of both livers were examined by $x$-ray energy dispersive spectroscopy (XES), and, in case 1 , by $x$-ray wavelength dispersive spectroscopy in a Jeol 35CF scanning electron microscope. Our methodology for $x$-ray spectroscopy of paraffin sections mounted on glass and perspex slides has been described in detail previously. ${ }^{4}$ Areas from both cases containing granular cells (described below), and adjacent tissues were analysed by XES at $25 \mathrm{kV}$ for periods of 100 seconds. Identical XES techniques were applied to paraffin sections of two normal livers and to sections of liver from cases of Wilson's disease, haemochromatosis, and cryptogenic cirrhosis. Samples of a tablet and a capsule of amiodarone were examined by XES under the same conditions. To enable transmission electron microscopy (TEM) to be performed on the granular cells part of a glass mounted paraffin section was reprocessed for TEM by the method of Bretschneider et al. ${ }^{5}$ It was viewed in a Jeol 2000 FX transmission electron microscope with XES attachment.

In an attempt to determine the precise histogenesis of the granular cells paraffin embedded sections of liver from both cases were stained by the peroxidase antiperoxidase (PAP) technique, following controlled trypsin digestion, using two monoclonal antibodies known to stain Kupffer cells and other macrophages, MAC $387^{6}$ and MT 1 (Eurodiagnostics), a monoclonal antibody to epithelial cytokeratin, CAM 5.2 (a gift of the ICRF), and a polyclonal antibody to a wider range of epithelial cytokeratins, keratin polyclonal PKE (Eurodiagnostics).

\section{Results}

The liver biopsy of case 1 showed preservation of the normal hepatic architecture. The main abnormality detected on light microscopy was the presence of focal collections of cells with swollen and granular cytoplasm (fig 1), which appeared yellow or brown in haematoxylin and eosin stained sections. These foci of granular cells were associated with a mild increase in chronic inflammatory cells, mainly lymphocytes, and were predominantly grouped in a perivenular location (fig 1). The granular pigment filled and distended 


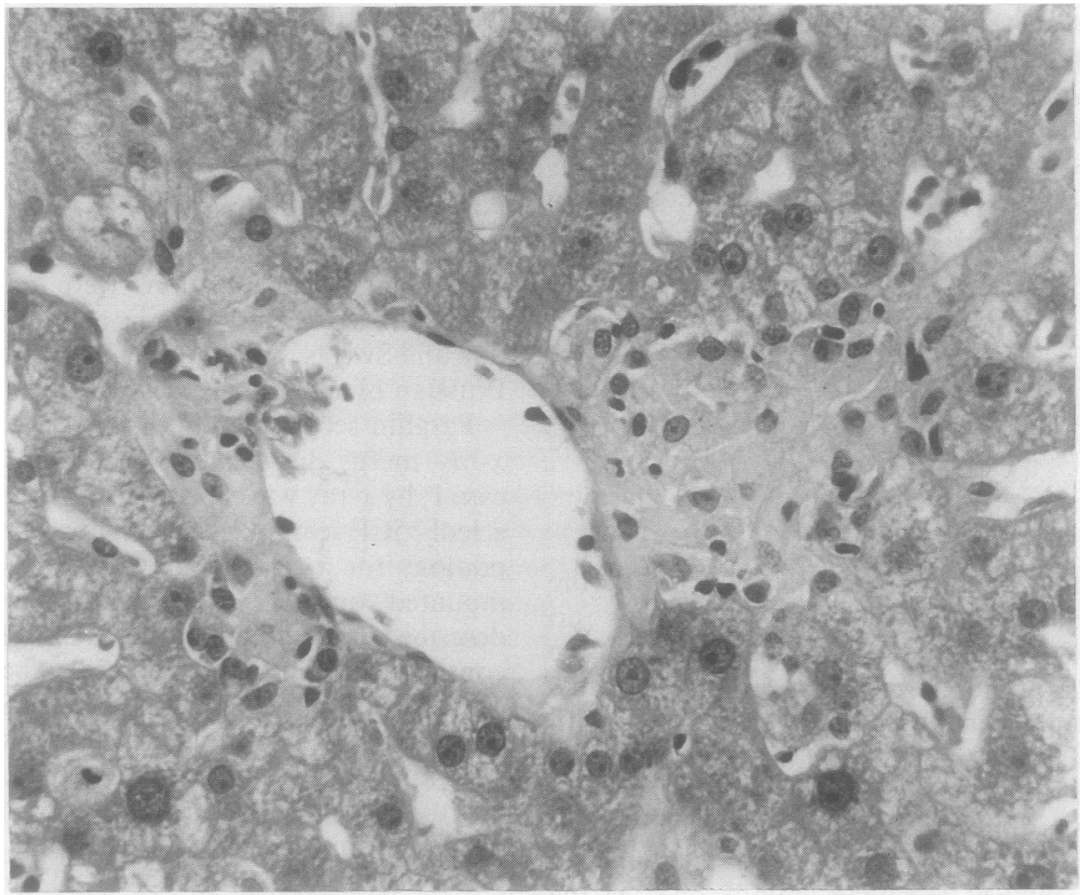

Fig 1 Liver biopsy specimen from case 1. Aggregates of pale, finely granular cells with scattered lymphocytes adjacent to central hepatic venule. (Haematoxylin and eosin.) $\times 250$.

the cell cytoplasm and varied from finely to coarsely granular, and closely resembled appearances seen in hereditary lipidoses. The granular cytoplasm was PAS positive, diastase resistant (fig $2 \mathrm{a}$ ) and was weakly stained with the Masson trichrome (fig $2 b$ ), unlike the adjacent normal hepatocytes which stained a deep blue or red. Thus the abnormal granular cells became much more obvious on diastase-PAS and Masson trichrome staining. There was no steatosis, cholestasis, Mallory's hyaline, or excess of stainable iron. The liver of case 2 showed a micronodular cirrhosis with extensive necrosis of the regenerative nodules. There was no noticeable inflammation, steatosis, or Mallory's hyaline. Within the fibrous scars, foci of cells with swollen granular cytoplasm, similar to those of case 1 , were seen. The cytoplasm of these cells was PAS positive diastase resistant and stained only weakly with the trichrome stain. In both cases the macrophage markers (MAC 387 and MT 1) stained Kupffer cells and other macrophages but not the granular cells or hepatocytes. The epithelial marker keratin polyclonal PKE, stained bile duct epithelium but no other cells. CAM 5.2 stained bile duct epithelium and hepatocytes but not the granular cells or Kupffer cells.
TEM showed that the granularity of the swollen cells in case 1 was due to lysosomal inclusion bodies? (fig 3). Their size varied from 0.4 to $2.4 \mu \mathrm{m}$. Although the preservation of the tissue was not optimal because of prior formalin fixation and paraffin wax embedding, a lamellar structure was identified in a small proportion of the inclusions. None of the adja- $\frac{O}{3}$ cent and histologically normal hepatocytes contained $\mathrm{O}$ lysosomal inclusion bodies.

XES on the paraffin sections in the scanning elec- $-\frac{D}{2}$ tron microscope gave the following results. The cytoplasm of all of the granular cells examined in both $N$ cases always produced a strong signal for iodine. Non iodine could be detected in adjacent hepatocytes, N Kupffer cells, or connective tissue. The only other ele- $\sigma$ ments detected were traces of $\mathrm{P}, \mathrm{S}, \mathrm{Cl}, \mathrm{Ca}$ and $\mathrm{Fe},<$ which were present in the granular cells and in the adjacent cells and tissues, although there were more of these elements in the granular cells. No iodine was ${ }_{7}$ detected in any of the control sections; iron was readily detected in the case of haemochromatosis and $\vec{\Phi}$ copper in the case of Wilson's disease. X-ray wave- $\frac{?}{\mathbb{D}}$ length analysis of the granular cells of case 10 confirmed the presence of iodine. XES performed in the transmission electron microscope confirmed that 8 


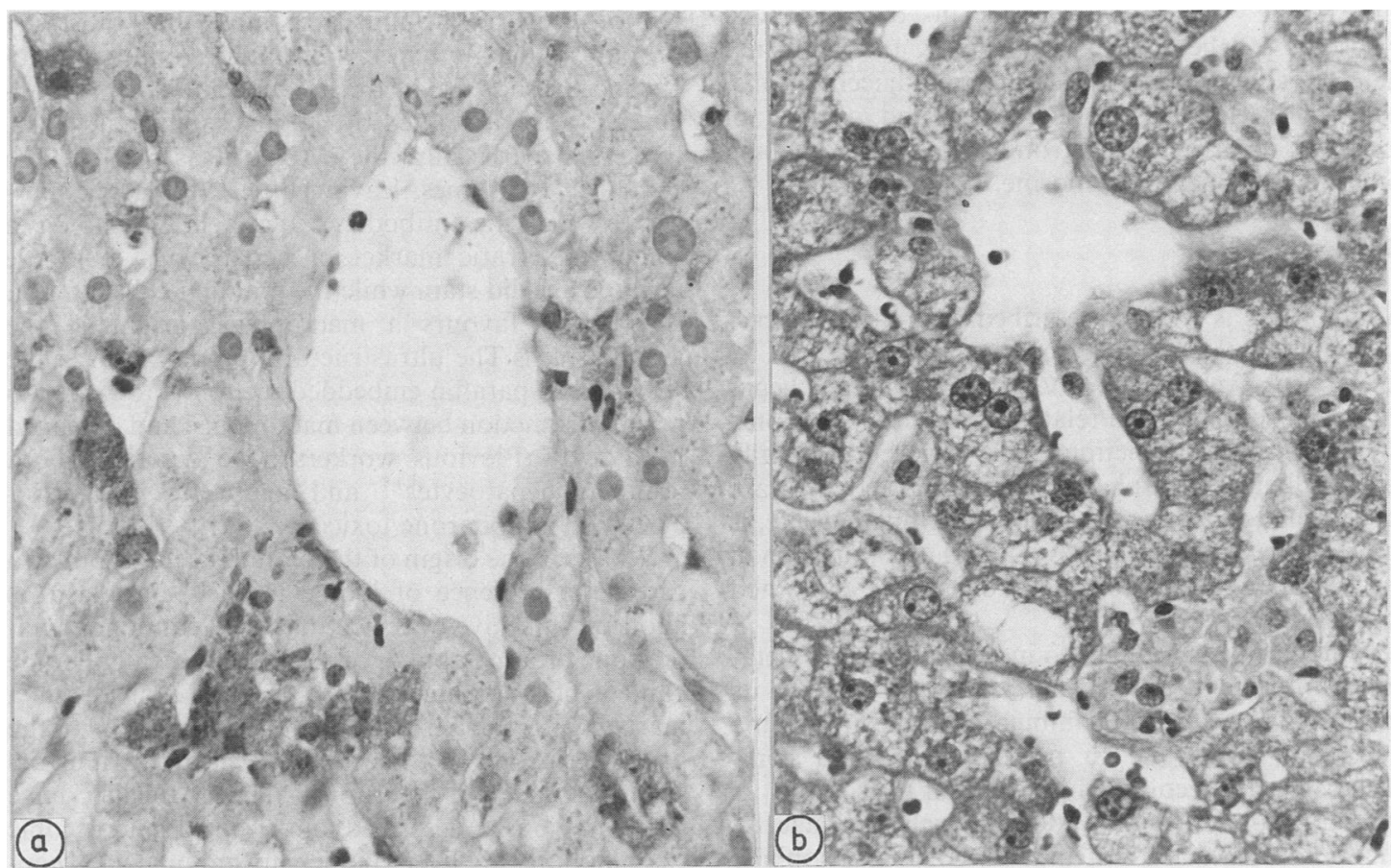

Fig 2 (a) Groups of dark granular cells near central hepatic venule (PAS post-diastase). (b) One group of granular cells below central hepatic venule contrasting with darker staining normal hepatocytes. (Masson trichrome.) $\times 425$.

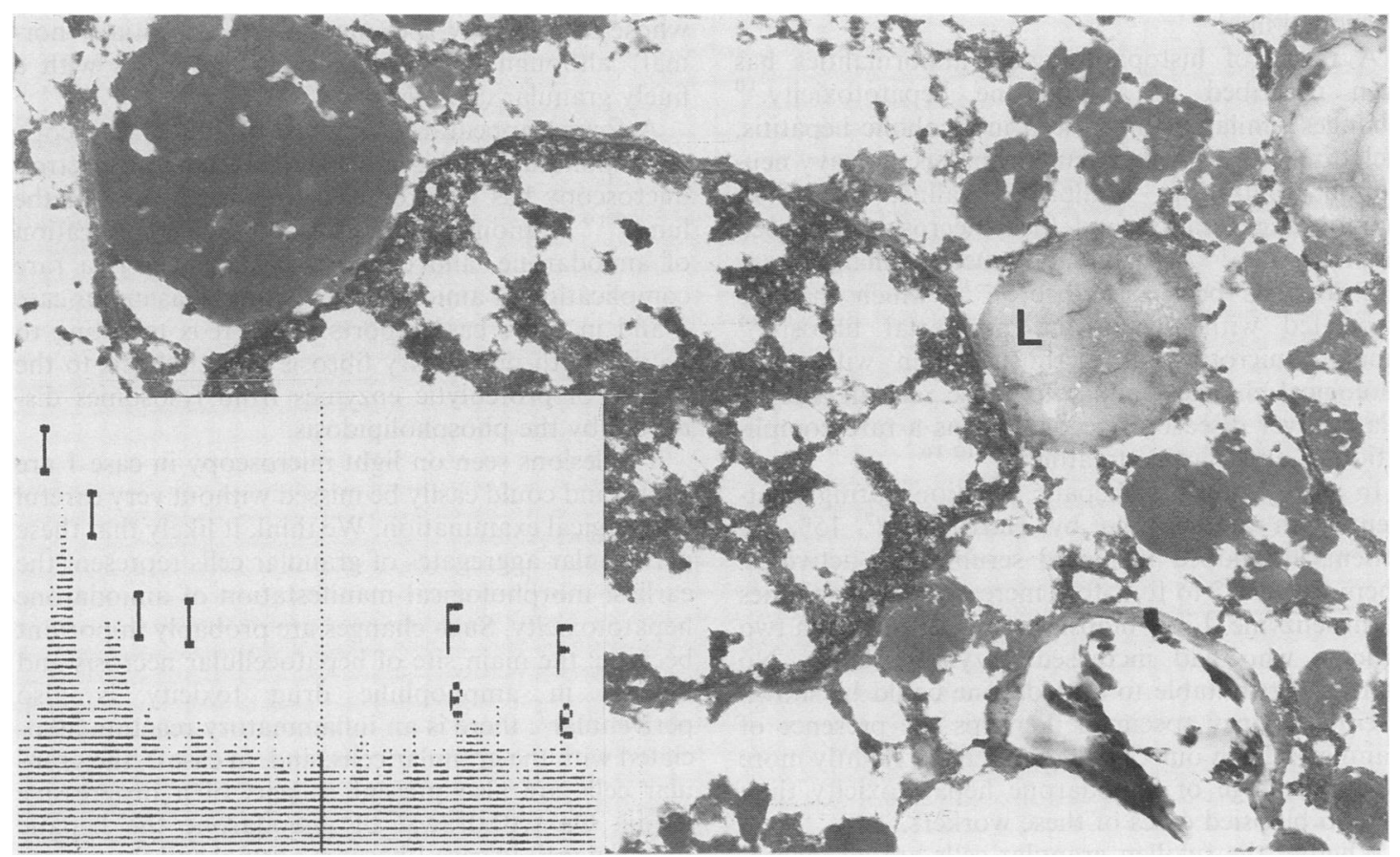

Fig 3 Transmission electron micrograph of reprocessed paraffin section, hence poor preservation. Lysosomes of variable appearance and part of nucleus shown. Inset is part of XES spectrum of lysosome $(L)$, showing peaks for iodine and traces of iron. Only other elements detected originated in stains and instrument. $\times 14000$. 
the iodine was only present in the distended lysosomes of the granular cells (fig 3), and it was not detected elsewhere in these cells or in adjacent normal cells. XES analysis of the tablet and capsule of amiodarone produced a strong signal for iodine and only a weak signal for chlorine.

\section{Discussion}

Amiodarone is widely prescribed for refractory cardiac arrhythmias. Its use, however, is complicated by toxicity in several organs. Clinically apparent hepatotoxicity seems to be a relatively rare complication. ${ }^{2}$ A change in liver function tests, however, especially raised alkaline phosphatase and AST activities are more common. ${ }^{2}$ The drug has a very long half life, is known to be stored in the liver, ${ }^{7}$ and accumulation in the liver may result in increased density on computed tomography scan. ${ }^{8}$

Amiodarone is thought to induce a systemic metabolic abnormality in lysosomes, probably due to its amphophilic nature. ${ }^{9}$ It combines with the lipid of the lysosomal membrane to produce drug-lipid complexes that are recognised by electron microscopy as the lamellar lysosomal bodies which characterise amiodarone toxicity and that of other amphophilic drugs. ${ }^{3}$ These lamellar bodies are lysosomes greatly expanded by the phospholipidosis produced by the combination of the amphophilic drug and the lysosomal lipid. ${ }^{3}$

A range of histopathological abnormalities has been described in amiodarone hepatotoxicity. ${ }^{10}$ Changes similar to those seen in alcoholic hepatitis, including steatosis, perivenular fibrosis, a heavy neutrophil infiltration, Mallory's hyaline, focal ballooning degeneration and focal necrosis have been reported. ${ }^{31-13}$ A portal mononuclear inflammatory infiltrate has been described, ${ }^{13-15}$ which may be associated with portal and periportal fibrosis. ${ }^{15}$ Finally, micronodular cirrhosis, often with morphological changes similar to those seen in alcohol related liver disease, is recognised as a rare complication of amiodarone treatment. ${ }^{31216}$

In a large study of hepatic function during treatment with amiodarone by Harris et al $15 \%$ of patients developed increased serum AST activities. There was a 1.5 to four-fold increase in the activities of this enzyme. Liver biopsies were performed in two patients who had increased enzyme activity. No changes attributable to amiodarone could be shown in either biopsy specimen. Perhaps the presence of granular cells in our case 1 represents a slightly more advanced stage of amiodarone hepatotoxicity than the two biopsied cases of these workers.

Whether the swollen granular cells are all macrophages or damaged hepatocytes remains uncertain.

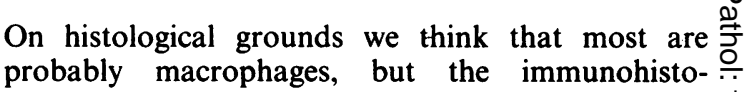
chemical results are inconclusive. Though the granu- $\overrightarrow{\overrightarrow{\mid}}$ lar cells are not stained by the macrophage markers, $\frac{\sigma}{0}$ this could be because the cytoplasm is packed with $\frac{\mathrm{C}}{0}$ expanded lysosomes, leaving little cytoplasmic anti- $\frac{\bar{\sigma}}{\omega}$. gen to which the antibodies can attach. CAM $5 \cdot 2$ is a $\vec{\nabla}$ somewhat erratic marker of hepatocytes, but here $\varrho$ hepatocytes did stain while the granular cells did not. This result favours a macrophage origin of the $\overrightarrow{0}$ granular cells. The ultrastructural preservation in the reprocessed paraffin embedded section is too poor to $\vec{\omega}$ permit distinction between macrophage and damaged hepatocyte. Previous workers have described both granular hepatocytes ${ }^{17}$ and aggregates of histio- $\frac{f}{0}$ cytes $^{13}$ in amiodarone toxicity.

Whatever the origin of the granular cells in our two $\stackrel{\oplus}{\vec{\infty}}$ cases, the presence of iodine in their cytoplasm is good presumptive evidence that they contained ami- $\triangle$ odarone or a metabolite of the drug. The high iodine $\vec{\nabla}$ content of amiodarone has been used clinically as a marker for the drug, and it is the iodine moiety of the drug that is known to accumulate in the various tis- $\vec{\oplus}$ sues. ${ }^{18}$ Lysosomal inclusions, mainly lamellar, have . been previously described on electron microscopic $\square$ examination of liver tissue in amiodarone heptotoxicity, ${ }^{39111315}$ but they have not been analysed by XES. Varma et al $^{17}$ found such lysosomal bodies in two patients with mild increases in AST activity $\stackrel{\square}{\square}$ whose liver biopsy specimens were "essentially nor- $\overrightarrow{\vec{F}}$ mal" although they do describe heptocytes with a $\frac{\circ}{3}$ finely granular cytoplasm.

A foamy appearance of cells on light microscopy corresponding to the lamellar inclusions at electron microscopy has been described in the alveoli of the ? lung. ${ }^{919}$ Pulmonary fibrosis is a known complication of amiodarone, and cirrhosis of the liver is a rare complication of amiodarone treatment, as in our case 2 and in other case reports. ${ }^{31216}$ It is tempting to ascribe both pulmonary fibrosis and cirrhosis to the 을 release of proteolytic enzymes from lysosomes dis- $\rightarrow$ rupted by the phospholipidosis.

The lesions seen on light microscopy in case 1 are $\bar{N}$ subtle and could easily be missed without very careful histological examination. We think it likely that these $N$ perivenular aggregates of granular cells represent the earliest morphological manifestation of amiodarone $\bar{Q}$ hepatotoxicity. Such changes are probably important 0 because: the main site of hepatocellular necrosis and $\Phi$ fibrosis in amphophilic drug toxicity is also $\stackrel{\circ}{+}$ perivenular ${ }^{7}$; there is an inflammatory reaction asso- 0 ciated with the granular cells; and, in case 2 , the gran- $\frac{\vec{D}}{\Phi}$ ular cells are also present as part of a progressive $\stackrel{\Phi}{\Phi}$ lesion. On withdrawal of amiodarone the hepatic $\mathbb{Q}$ function tests in case 1 became normal and the patient $\overline{0}$ recovered completely. This suggests that the mor- 
phological lesion we have described is reversible on stopping the drug and may not progress. We believe that the small, predominantly perivenular, foci of granular cells may be a useful histopathological marker of early amiodarone hepatotoxicity.

We are grateful to Dr RAJ Spurrell for permission to report the clinical details of case 2. We thank $\mathrm{Mr}$ Clive Wells and Mr Robert Freeman for technical expertise. The North East Thames Regional Health Authority, The Wellcome Trust, and St Bartholomew's Hospital Joint Research Board financed our analytical apparatus.

\section{References}

1 Krikler DM. Amiodarone: the background. In: Krikler DM, McKenna WJ, Chamberlain DA, eds. Amiodarone and arrhythmias. Oxford: Pergamon Press, 1983:3-4.

2 Harris L. Unwanted effects of long-term amiodarone treatment. In: Krikler DM, McKenna WJ, Chamberlain DA, eds. Amiodarone and arrhythmias. Oxford: Pergamon Press, 1983:61-8.

3 Poucell S, Ireton J, Valencia-Mayoral P, et al. Amiodaroneassociated phospholipidosis and fibrosis of the liver. Light, immunohistochemical and electron microscopic studies. Gastroenterology 1984;86:926-36.

4 Crocker PR, Toulson E, Levison DA. Particles in paraffin sections demonstrated by the backscattered electron image (BEI). Micron 1982;13:437-46.

5 Bretschneider A, Burns W, Morrison A. "Pop-off" technic. The ultrastructure of paraffin-embedded sections. Am J Clin Pathol 1981;76:450-3.

6 Flavell DJ, Jones DB, Wright DH. Identification of tissue histiocytes on paraffin sections by a new monoclonal antibody. J Histochem Cytochem (in press)

7 Harris L, McKenna WJ, Rowland E, Holt DW, Storey GCA,
Krikler DM. Side effects of long-term amiodarone therapy. Circulation 1983;67:45-51.

8 Goldman IS, Winkler ML, Raper SE, et al. Increased hepatic density and phospholipidosis due to amiodarone. Am J Roentgenol 1985; 144:541-6.

9 Dake MD, Madison JM, Montgomery CK, et al. Electron microscopic demonstration of lysosomal inclusion bodies in lung, liver, lymph nodes, and blood leukocytes of patients with amiodarone pulmonary toxicity. Am J Med 1985;78:506-12.

10 Sherlock S. The spectrum of hepatotoxicity due to drugs. Lancet 1986;ii:440-4.

11 Simon JB, Manley PN, Brien JF, Armstrong PW. Amiodarone hepatotoxicity simulating alcoholic liver disease. $N$ Engl $J$ Med 1984;311:167-72.

12 Tordjman K, Katz I, Bursztyn M, Rosenthal T. Amiodarone and the liver. Ann Intern Med 1985;102:411-2.

13 Rigas B, Rosenfeld LE, Barwick KW, et al. Amiodarone hepatotoxicity. A clinicopathological study of five patients. Ann Intern Med 1986;104:348-51.

14 Yagupsky P, Gazala E, Sofer S, Maor E, Abarbanel J. Fatal hepatic failure and encephalopathy associated with amiodarone therapy. J Pediatrics 1985;107:967-70.

15 Capron-Chivrac D, Reix N, Quénum C, Capron J-P. Amiodarone hepatopathy. Study of a case and review of the literature. Gastroenterol Clin Biol 1985;9:535-9.

16 Lim PK, Trewby PN, Storey GCA, Holt DW. Neuropathy and fatal hepatitis in a patient receiving amiodarone. $\mathrm{Br} \mathrm{Med} \mathrm{J}$ 1984;288:1638-9.

17 Varma RR, Troup PJ, Komorowski RA, Sarna T. Clinical and morphological effects of amiodarone on the liver. Gastroenterology 1985;88:1091-2.

18 Broekhuysen J. Pharmacology of amiodarone-history and prospects. In: Krikler DM, McKenna WJ, Chamberlain DA, eds. Amiodarone and arrhythmias. Oxford: Pergamon Press, 1983:5-10.

19 Costa-Jussa FR, Corrin B, Jacobs JM. Amiodarone lung toxicity: a human and experimental study. $J$ Pathol 1984;143:73-9.

Requests for reprints to: Dr NA Shepherd, Department of Histopathology, St Bartholomew's Hospital, West Smithfield, London EClA 7BE, England. 